\title{
Design of a Variable-Geometry Suspension System to Enhance Road Stability
}

\author{
Balázs Németh, Balázs Varga and Péter Gáspár
}

\begin{abstract}
The paper proposes the design of a variablegeometry suspension system, which affects both steering by generating additional steering angle and wheel tilting by modifying the camber angle. The control system must guarantee various performances such as trajectory tracking, the reduction of the chassis roll angle and the minimization of half-track change. The control design is based on a two-step procedure. The performance specifications are met by a high-level control, in which the control input is a required signal. In the lowlevel control the actuator must track this signal by adjusting its current signal. The advantage of this modular design is that the actuator level does not affect the design of a highlevel control. The operation of the designed control system is illustrated through a simulation example.
\end{abstract}

\section{INTRODUCTION}

The variable-geometry suspension system provides new possibilities in driver assistance systems. An analysis shows that this system affects both steering by generating additional steering angle and wheel tilting by modifying the camber angle. The advantages of the variable-geometry suspension are the simple structure, low energy consumption and low cost, see [1]. In this paper the combination of wheel tilting and steering is proposed.

Several papers for various kinematic models of suspension systems have been published, see [2], [3]. Seeking to meet the performance requirements often leads to conflicts and requires a compromise considering the kinematic and dynamic properties, see [4]. The vehicle handling characteristics based on a variable roll center suspension were presented by [5]. It has also been shown that the control design is in interaction with the construction of the system, see [6].

In the control design different control specifications must be guaranteed such as trajectory tracking, the reduction of the chassis roll angle and the minimization of half-track change. A weighting strategy is applied to achieve a balance between performance specifications. Moreover, the actuator dynamics is also built into the control design. The direct inclusion of the actuator dynamics in the vehicle dynamics leads to a high-complexity model. If the control design were carried out on the basis of this model, this might lead to numerical problems due to the increased complexity.

The research has been conducted as part of the project TÁMOP-4.2.2.A11/1/KONV-2012-0012: Basic research for the development of hybrid and electric vehicles. The Project is supported by the Hungarian Government and co-financed by the European Social Fund.

The authors are with Systems and Control Laboratory, Institute for Computer Science and Control, Hungarian Academy of Sciences, Kende u. 13-17, H-1111 Budapest, Hungary. E-mail: [ bnemeth; bvarga; gaspar] @sztaki.mta.hu
Thus, in the paper a two-step procedure is proposed for the control design. In the design of a high-level control the vehicle model containing both the steering angle and the camber angle is considered where performance specifications must be guaranteed and the control input is the required signal. In this step the uncertainties of the model are also considered and a weighting strategy is applied to create a balance between performance specifications. The required signal must be tracked by the low-level actuator control by adjusting its current signal. The advantage of the two-step procedure proposed in this paper is that the control of the variable-geometry suspension system and the control of its actuator are handled in two independent control design steps.

The reason for the separation is that the actuator level does not affect the design of a high-level control, thus the two different control design tasks are performed independently. In the high-level control a parameter-dependent LPV method while in the low-level control the $\mathcal{H}_{\infty} / \mu$ method is applied in the design.

This paper is organized as follows. Section II presents the dynamic interconnection between the steering angle and the camber angle. Section III proposes the control design of the variable-geometry suspension system, in which several performances must be guaranteed simultaneously. Section IV presents the actuator dynamics and the design of its robust control. Section V illustrates the operation of the control system through a simulation example. Finally, Section VI summarizes the contributions.

\section{DYNAMIC EFFECTS OF THE VARIABLE-GEOMETRY SUSPENSION SYSTEM}

The variable-geometry suspension system affects both the position and the orientation of the front wheels. In the aspect of a driver assistance system, steering angle $\delta$ and camber angle of the front wheels $\gamma$ are relevant [6]. $\delta$, which is the angle between the direction of the front wheel and that of the vehicle, has two components, i.e., $\delta=\delta_{d}+\delta_{c} . \delta_{d}$ is performed by the driver, while $\delta_{c}$ is the control signal generated by the variable-geometry suspension system. In the following the dynamic effects of the variable-geometry suspension system on both steering and wheel tilting are presented.

A bicycle model of the vehicle used in the description of vehicle dynamics is extended by the wheel camber angle. The Magic form of the tire dynamics describes the effects on the steering angle, the camber angle and the lateral tyre forces $\left(F_{y}\right)$, see [7]. Although it results in an accurate approximation of the lateral tire forces, in control design 
tasks a simplified form is used. Based on the Magic form [8] proposes a linear relationship between $\delta_{c}, \gamma$ and the lateral tire forces. This lateral tire model in the direction of the wheel-ground contact is approximated $F_{y f}=C_{1} \alpha_{f}+C_{1, \gamma} \gamma$ and $F_{y r}=C_{2} \alpha_{r}$, where $\gamma$ is the wheel camber angle, $C_{1}, C_{2}$ are cornering stiffnesses at the front and the rear and $C_{1, \gamma}$ is the wheel camber stiffness.

The vehicle is moving along the road, where both the longitudinal and the lateral dynamics must be taken into consideration as shown in Figure 1.

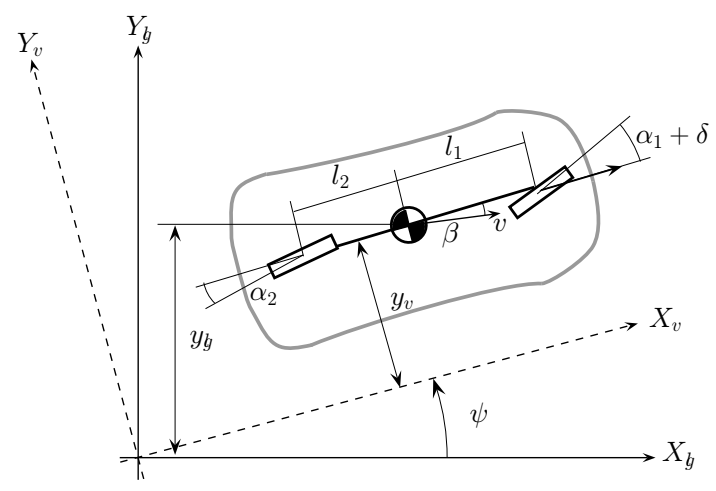

Fig. 1. Lateral model of the vehicle

The bicycle model is as follows:

$$
\begin{aligned}
J \ddot{\psi} & =C_{1} l_{1} \alpha_{f}-C_{2} l_{2} \alpha_{r}-C_{1, \gamma} l_{1} \gamma \\
m v(\dot{\psi}+\dot{\beta}) & =C_{1} \alpha_{f}+C_{2} \alpha_{r}+C_{1, \gamma} \gamma
\end{aligned}
$$

where $J$ is the yaw inertia of the vehicle, $l_{1}$ and $l_{2}$ are geometric parameters, $\psi$ is the yaw of the vehicle, $\beta$ is the side-slip angle of the vehicle. and $v$ is velocity. Moreover, $\alpha_{f}=\delta_{d}+\delta_{c}-\beta-l_{1} \dot{\psi} / v$ and $\alpha_{r}=-\beta+l_{2} \dot{\psi} / v$ are the tire side slip angles at the front and rear, respectively.

(1) and (2) show that three signals have effects on lateral dynamics: $\delta_{d}, \delta_{c}$ and $\gamma . \delta_{d}$ is performed by the driver, while the other two signals are control signals of the driver assistance system. However, $\delta_{c}$ and $\gamma$ are not independent of each other. Both of them depend on the lateral displacement of the actuator of the variable-geometry suspension system $a_{y}$, i.e., $\delta_{c}=\delta_{c}\left(a_{y}\right), \gamma=\gamma\left(a_{y}\right)$. The actuator of the variablegeometry suspension system is illustrated in Figure 2.

The state space representation of the variable-geometry suspension system is the following:

$$
\dot{x}=A(\rho) x+B_{1}(\rho) w+B_{2}(\rho) u
$$

where the state vector is $x=\left[\begin{array}{ll}\dot{\psi} & \beta\end{array}\right]^{T}$, the disturbance is $w=\delta_{d} . u$ is the control signal, which depends on both $\delta_{c}$ and $\gamma$. Since these signals are the function of $a_{y}$, the control signal will be expressed by $u=a_{y}$. The system matrices depend on the velocity of the vehicle nonlinearly. Using a scheduling variable $\rho=v$ the nonlinear model is transformed into an LPV model.

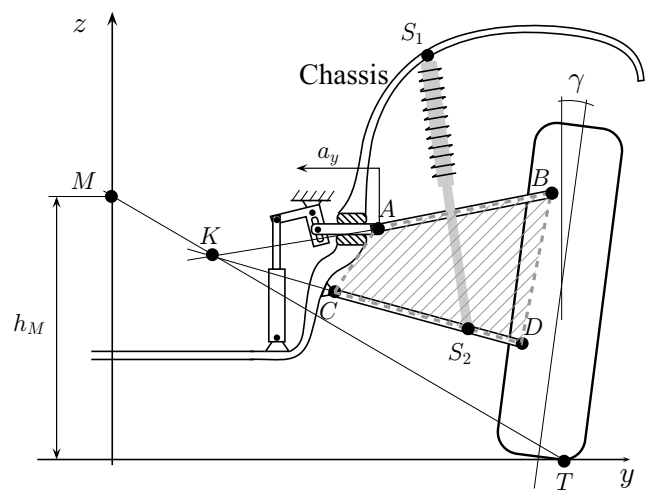

Fig. 2. Actuator of variable-geometry suspension

\section{DESIGN OF THE HIGH-LEVEL CONTROL}

The variable-geometry suspension system affects both steering by generating additional steering angle and wheel tilting by modifying the camber angle. This system assists the driver during maneuvers, i.e., trajectory tracking can be performed by modifying both the steering angle and the camber angle. Besides, the variable-geometry suspension has an effect on the chassis roll angle and the lateral movement of the tire-road contact. Consequently, several performance requirements must be defined, such as yaw-rate tracking, the roll angle, the half track change and the control input:

$$
z=\left[\begin{array}{llll}
z_{e_{\dot{\psi}}} & z_{\Delta h_{M}} & z_{\Delta B} & z_{a c t}
\end{array}\right]^{T}
$$

The goal of the control design is to guarantee performances simultaneously.

In the trajectory tracking control the vehicle must follow the reference yaw rate. The purpose is to minimize the difference between the actual yaw rate of the vehicle and the reference yaw rate:

$$
z_{e_{\psi}}=\left|\dot{\psi}_{\text {ref }}-\dot{\psi}\right| \rightarrow \min
$$

Note that the reference yaw rate represents the driver requirement, which depends on steering angle of the driver $\delta_{d}$.

The height of the roll center has an important role in the vertical dynamics of the vehicle as it determines the roll motion. A possible way to minimize the chassis roll angle is the minimization of the height of the roll center $h_{M}$. In this case the difference between the roll center and the center of gravity must be minimized:

$$
z_{\Delta h_{M}}=\left|h_{C G}-h_{M}\right| \rightarrow \min
$$

Note that the height of the roll center in steady state is determined by the suspension construction.

An important economy parameter is the half-track change $\Delta B=f\left(t_{z}, a_{y}\right)$. The lateral movement of the contact point is relevant from the aspect of tire wear, when the suspension moves up and down while the vehicle moves forward, see [9]. Thus, the unnecessary movements must be eliminated:

$$
z_{\Delta B}=|\Delta B| \rightarrow \min
$$


The control tasks should be achieved by as little control input as possible. Thus, the performance focuses on the minimization of the input displacement:

$$
z_{a c t}=\left|a_{y}\right| \rightarrow \min
$$

Moreover, during the control tasks it is necessary to prevent a large control input, which is the lateral movement of the suspension arm $a_{y}$, which is a construction limit.

The control design is based on the closed-loop interconnection structure of the system, see Figure 3. To emphasize the different importance of the performances simultaneously, weighting functions $W_{i}, i \in[1,4]$ are used. The control design is based on the performance weighting functions. If large weight is applied for the $z_{e_{\dot{\psi}}}$, the controlled system focuses on the trajectory tracking.

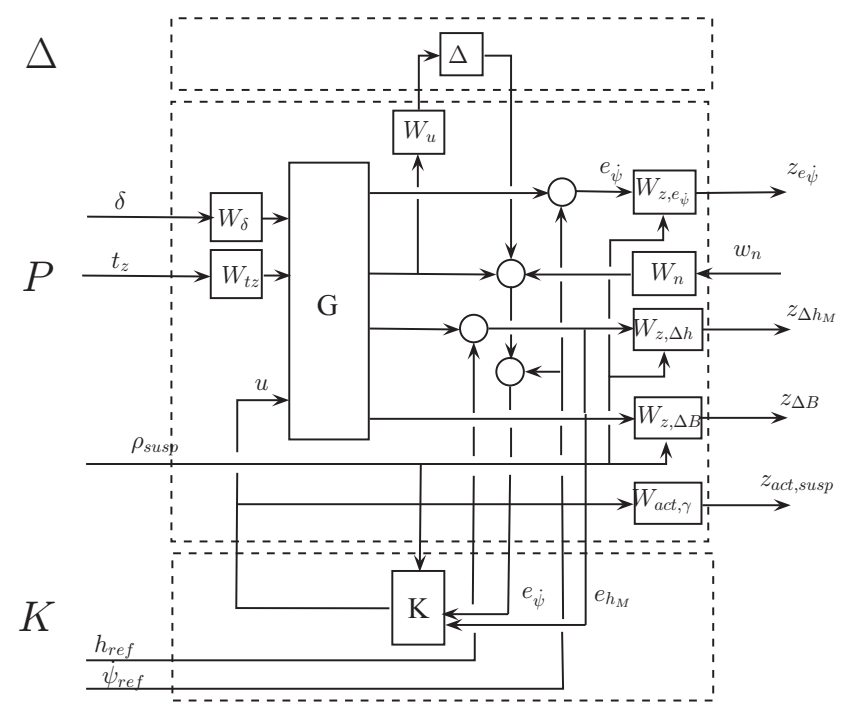

Fig. 3. Closed-loop interconnection structure

Using (3) and (4) the control design is based on the statespace representation of the system:

$$
\begin{aligned}
\dot{x} & =A(\rho) x+B_{1}(\rho) w+B_{2}(\rho) u \\
z & =C_{1}(\rho) x+D_{11}(\rho) w+D_{12}(\rho) u \\
y & =C_{2}(\rho) x+D_{21}(\rho) w
\end{aligned}
$$

where the the control signal is $u=a_{y}$ and the measured signal is the yaw rate: $y=\dot{\psi}$.

The control design of the variable-geometry suspension is based on the LPV method, which uses parameter-dependent Lyapunov functions, see [10], [11]. The quadratic LPV performance problem is to choose the parameter-varying controller in such a way that the resulting closed-loop system is quadratically stable and the induced $\mathcal{L}_{2}$ norm from the disturbance and the performances is less than a predefined value

$$
\inf _{K} \sup _{\Delta} \sup _{\|w\|_{2} \neq 0, w \in \mathcal{L}_{2}} \frac{\|z\|_{2}}{\|w\|_{2}} .
$$

where $w$ is the disturbance and $\Delta$ represents the unmodelled dynamics.

\section{Design of Robust CONTROL ON THE ACTUATOR LEVEL}

The intervention of variable-geometry suspension systems requires the realization of lateral motion $a_{y}$. In a real implementation it is realized using an electro-hydraulic actuator [12], [13] or an electric motor [1]. The purpose of the actuator is the realization of the desired $a_{y}$ motion, which leads to a positioning control on the actuator level. In the paper the electro-hydraulic construction is considered as the real actuator of the system. Several papers deal with the modeling and control of electro-hydraulic actuators, see e.g. [14], [15], [16]. The electro-hydraulic system consists of two main parts: a hydraulic cylinder and an electronicallycontrolled spool valve.

The electro-hydraulic actuator of the variable-geometry system is illustrated in Figure 5. Control input $a_{y}$ is realized by the displacement of hydraulic cylinder $x_{c}$, which is controlled by pressure difference $p_{L}$ between its chambers $V_{1}$ and $V_{2}$. The value and direction of the pressure difference are influenced by the displacement of the spool valve, which is controlled by the current of armatures. Thus, the physical input of the actuator is current $i$, while the output is the cylinder displacement $x_{c}$.

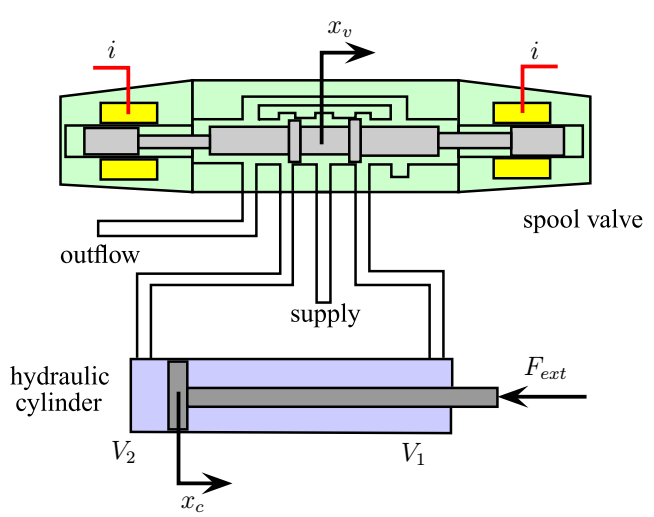

Fig. 4. Electro-hydraulic actuator

The pressures in the chambers depend on the flows of the circuits $Q_{1}, Q_{2}$. Because of the change of flow directions in the circuits, the hydraulic cylinder can be considered a switching system. However, at small $x_{c}$ the average flow of the system

$$
Q_{L}=C_{d} A\left(x_{v}\right) \sqrt{\frac{1}{\rho}\left(p_{s}-\frac{x_{v}}{\left|x_{v}\right|} p_{L}\right)}
$$

can be linearized around the center position of the cylinder by using the following equation [15]:

$$
Q_{L}=K_{q} x_{v}-K_{c} p_{L}
$$

where $K_{q}$ is the gain coefficient of the valve flow and $K_{c}$ is the pressure coefficient. The dynamics of the pressure difference is as follows [17]:

$$
\dot{p}_{L}=\frac{4 \beta_{e}}{V_{t}}\left(Q_{L}-A_{p} \dot{x}_{c}+c_{l 1} \dot{x}_{c}-c_{l 2} p_{L}\right)
$$


where $\beta_{e}$ is the effective bulk modulus, $V_{t}$ is the total volume under pressure, $A_{p}$ is the area of the piston, $c_{l 1}$ and $c_{l 2}$ are construction parameters.

Due to the pressure difference and the external load the position of the cylinder is determined by the motion equation of the piston:

$$
m_{c} \ddot{x}_{c}+d_{c} \dot{x}_{c}=A_{p} p_{L}+F_{\text {ext }},
$$

where $m_{c}$ is the masses of the piston and the mechanism between the piston and the suspension arm, $d_{c}$ is the damping constant of the system.

The electronically controlled spool valve is modeled as a second-order linear system, which creates linear dependence between current $i$ and spool displacement $x_{v}$, see [14]:

$$
\frac{1}{\omega_{v}^{2}} \ddot{x}_{v}+\frac{2 d_{v}}{\omega_{v}} \dot{x}_{v}+x_{v}=k_{v} i
$$

where $d_{v}$ is the valve damping coefficient, $\omega_{v}$ is the natural frequency of the valve. $k_{v}$ valve gain is formulated as $k_{v}=Q_{N} /\left(i_{\max } \sqrt{\Delta p_{N} / 2}\right)$, where $i_{\max }$ is the permitted maximum current, while $Q_{N}$ and $\Delta p_{N}$ are the flow and pressure drop at $i_{\max }$.

The dynamics of the electro-hydraulic actuator is described by the equations of hydraulic cylinder (15), (16) and (17). The actuator model is transformed into the following state-space representation:

$$
\dot{x}_{a c t}=A_{a c t} x_{a c t}+B_{a c t, 1} w_{a c t}+B_{a c t, 2} u_{a c t}
$$

The state vector of the actuator is $x_{a c t}=$ $\left[\begin{array}{lllll}\dot{x}_{v} & x_{v} & p_{L} & \dot{x}_{c} & x_{c}\end{array}\right]^{T}$, disturbance is $w_{\text {act }}=F_{\text {ext }}$ and control input is $u_{\text {act }}=i$.

The electro-hydraulic actuator model is based on the linearization of the hydraulic cylinder. The model contains several parameters. There are physical parameters whose values are known from the operation of the system, e.g., $\beta_{e}$ depends on the pressure of chambers. There are parameters whose values are yielded by an identification procedure, e.g., $K_{q}$ and $K_{c}$ in (14). Moreover, the model to be used in the control design contains components the properties of which are uncertain. The uncertainty of the model is caused by neglected dynamics, uncertain components, inadequate knowledge of components, or alteration of their behavior due to changes in operating conditions. Thus, in the control design the parameter uncertainties must be considered.

Uncertainty is taken into consideration in an unstructured way in the $\mathcal{H}_{\infty}$ control synthesis, thus the design process yields a conservative controller. In the complex $\mathcal{H}_{\infty} / \mu$ method, the structure of uncertainties is represented by a diagonal structure with full or scalar complex blocks. In practice, usually parametric uncertainties occur, thus they should be represented by repeated real blocks. In the real $\mathcal{H}_{\infty} / \mu$ method, the structure of uncertainties is represented by both complex and real blocks, see [18], [19]. In this paper the $\mathcal{H}_{\infty} / \mu$ control design method is applied to guarantee the stability of the system against parameter variations.

The most important parametric uncertainties of the system are the variation of $K_{q}, K_{c}$ and $\beta_{e}$, see [15]. In (14) and (15), the parameters are assumed to be uncertain, with a nominal value and a range of possible variations. All uncertainty parameters are written in the lower linear fractional transformation (LFT) form. As an example value $\beta_{e}$ is expressed as follows:

$$
\beta_{e}=\bar{\beta}_{e}\left(1+d_{e} \delta_{e}\right)=\mathcal{F}_{l}\left(\left[\begin{array}{cc}
\bar{\beta}_{e} & 1 \\
d_{e} \bar{\beta}_{e} & 0
\end{array}\right], \delta_{e}\right)=\mathcal{F}_{l}\left(M_{e}, \delta_{e}\right)
$$

In the LFT structure the relationship between the output and the input of the block $M_{e}$ is $\tilde{y}_{e}=\bar{\beta}_{e} \tilde{u}_{e}+u_{e}$, while the uncertainty block $\delta_{e}$ is pulled out of the equation. $\bar{K}_{q}, \bar{K}_{c}, \bar{\beta}_{e}$ denote the nominal values of the parameters, $d_{q}, d_{c}, d_{e}$ are scalars, which represent the percentage of variation that is allowed for a given parameter around its nominal value and $-1 \leq \delta_{q}, \delta_{c}, \delta_{e} \leq 1$ determines the actual parameter deviation. In the formulation of parametric uncertainties, all of the $\delta_{i}, i \in(q, c, e)$ blocks must be pulled out of the motion equations.

The formulated $\tilde{y}_{i}$ outputs are used in (14) and (15) to express the parametric uncertainty of the system as follows:

$$
\begin{aligned}
\dot{p}_{L} & =\frac{4 \bar{\beta}_{e}}{V_{t}}\left(\bar{K}_{q} x_{v}-\bar{K}_{c} p_{L}-A_{p} \dot{x}_{c}+c_{l 1} \dot{x}_{c}-c_{l 2} p_{L}\right)+ \\
& +\frac{4}{V_{t}}\left(\bar{\beta}_{e} u_{q}-\bar{\beta}_{e} u_{c}+u_{e}\right)
\end{aligned}
$$

The uncertainties of the system are involved as disturbances in the state-space representation. Thus, (18) is modified as:

$$
\dot{x}_{u a}=A_{u a} x_{u a}+B_{u a, 1} w_{u a}+B_{u a, 2} u_{u a}
$$

where the state vector, the disturbance and the control input are $x_{u a}=\left[\begin{array}{lllll}\dot{x}_{v} & x_{v} & p_{L} & \dot{x}_{c} & x_{c}\end{array}\right]^{T}, w_{u a}=$ $\left[\begin{array}{llll}F_{\text {ext }} & u_{q} & u_{c} & u_{e}\end{array}\right]^{T}$ and $u_{u a}=i$, respectively.

The goal of the positioning control of the electro-hydraulic system is to move the piston $x_{c}$ to a reference value $x_{c, r e f}$. Thus, the following tracking error minimization must be guaranteed:

$$
z_{1}=x_{c, \text { ref }}-x_{c} ; \quad\left|z_{1}\right| \rightarrow \min !
$$

Besides, the control input of the system must be minimized because of the permitted maximum value of current $i$ :

$$
z_{2}=i ; \quad\left|z_{2}\right| \rightarrow \min !
$$

The performance vector of the system is formed as $z_{u a}=$ $\left[\begin{array}{ll}z_{1} & z_{2}\end{array}\right]^{T}$. The designed robust controller requires the measurement of the piston position $y_{u a}=x_{c}$. The performance and measurement equations are formulated as:

$$
\begin{aligned}
& z_{u a}=C_{u a, 1} x_{u a}+D_{u a} u_{u a} \\
& y_{u a}=C_{u a, 2} x_{u a}
\end{aligned}
$$

(20), (23) and (24) describe the dynamics, performances and measurements of the uncertain electro-hydraulic actuator, respectively.

The reference motion of piston $x_{c, r e f}$ is determined by the required control input of the upper-level control $a_{y}$. The relationship between reference $x_{c, r e f}$ and lateral displacement $a_{y}$ is formed: $x_{c, r e f}=f\left(a_{y}\right)$. It depends on the mechanism, 
which connects the cylinder and the upper-arm, while $f$ is a static nonlinear function, thus it can be implemented as a look-up table in the control system, see e.g., Figure 5.

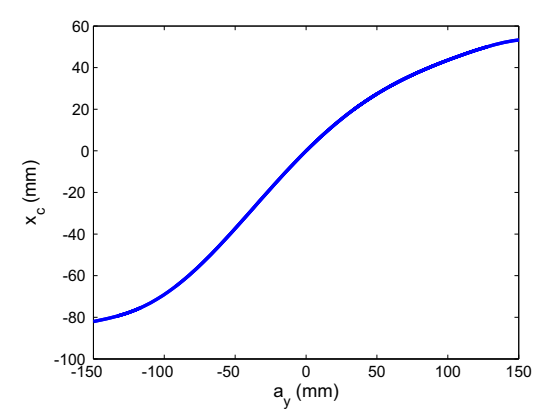

Fig. 5. Static map between $a_{y}$ and $x_{c}$

The aim of control design is to guarantee stability and the performances of the system against disturbance $F_{\text {ext }}$ while uncertainties $d_{q}, d_{c}, d_{e}$ are taken into consideration. The robust $\mathcal{H}_{\infty} / \mu$ control design method is able to handle performance criteria, disturbance rejection and parametric uncertainties together.

The balance between performances is guaranteed by the scaling of performances using weighting functions. $W_{z, 1}=$ $\lambda\left(\epsilon_{1} s+1\right) /\left(T_{1} s+1\right)$ represents the frequency-dependent weight of positioning error minimization. The appropriate choice of $\epsilon_{1}$ and $T_{1}$ ensures that the error is reduced to $1 / \lambda$ at low-frequencies. $W_{z, 2}=1 / i_{\max }$ is related to $z_{2}$. $\Delta$ incorporates the parametric uncertainties of the system, $W_{\text {ext }}$ and $W_{\text {ref }}$ scales disturbance and reference signal of the system. In the robust $\mathcal{H}_{\infty} / \mu$ control design the controller synthesis problem is the following. Find a controller $K$ such that

$$
\mu_{\tilde{\Delta}}(M(i \omega)) \leq 1, \forall \omega \Leftrightarrow \min _{K \in \mathcal{K}_{s t a b}}\left[\max _{\omega} \mu(M(i \omega))\right]
$$

where $\mu$ is the function of the structured singular value of the system $M(i \omega)$ with a given uncertainty set $\tilde{\Delta}=$ $\operatorname{diag}\left[\Delta_{r}, \Delta_{m}, \Delta_{p}\right] . \Delta_{r}$ represents the parametric uncertainties, $\Delta_{m}$ describes the unmodelled dynamics and $\Delta_{p}$ is a fictitious uncertainty block, which incorporates the performance objectives into the $\mu$ framework.

The optimization problem can be solved in an iterative way by using a two-parameter minimization in a sequential fashion: first minimizing over control $\mathrm{K}$ with scaling $\mathrm{D}$ fixed, then minimizing over $\mathrm{D}$ with $\mathrm{K}$ fixed, and so on. The control design is a standard $\mathcal{H}_{\infty}$ optimization problem, while finding $\mathrm{D}$ is a standard convex optimization problem. The optimization problems are intractable in most cases, but an ad hoc algorithm known as $D-K$ iteration has been found, see [18].

\section{Simulation example}

In the simulation example the operation of the variablegeometry suspension system is illustrated through a typical medium-size car. The control design of the suspension system is performed by the Matlab/Simulink software, while the verification of the controller is performed by the CarSim software, which is able to simulate vehicle dynamics with high accuracy.

$K_{z}$ is selected at different values, i.e., $K_{z}=100 \mathrm{~mm}$ and $K_{z}=600 \mathrm{~mm}$. Figure 6 shows the results of simulations. The operations of three systems are compared. The uncontrolled system is illustrated by solid blue line, the controlled system, in which $K_{z}=100 \mathrm{~mm}$, is illustrated by dashed green line, while the control system, in which $K_{z}=600$ $m m$, is illustrated by dash-dotted red line.

Figure 6(a) illustrates the course of the vehicle. The vehicle is driven along the course at $95 \mathrm{~km} / \mathrm{h}$ velocity, which can be dangerous for the vehicle in the middle sections of the road because of sharp bends. Figure 6(b) shows that the lateral error of the uncontrolled vehicle is unacceptable. There are sections in which the deviation from the centerline

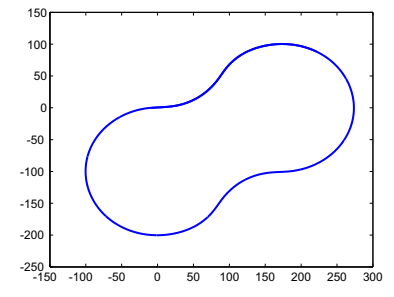

(a) Course of vehicles

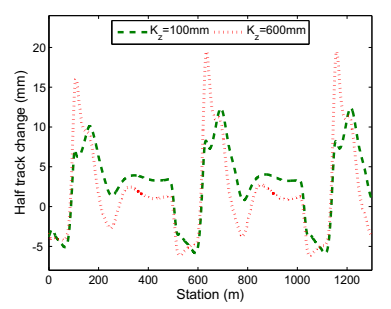

(c) Half-track change

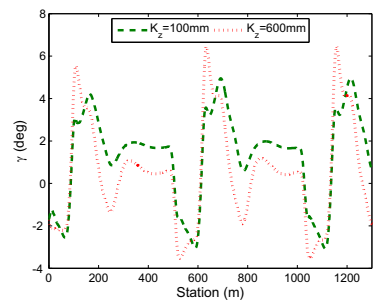

(e) Front left wheel camber

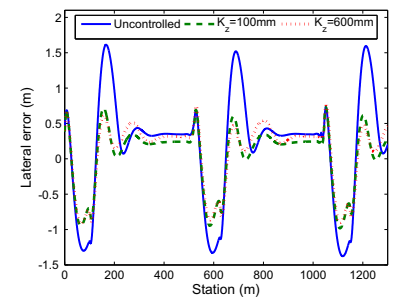

(b) Lateral error

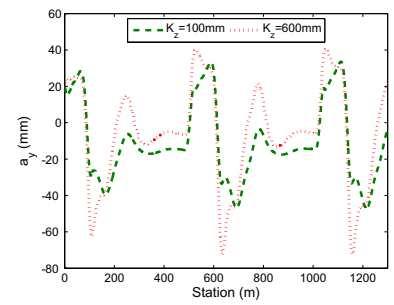

(d) Control actuation

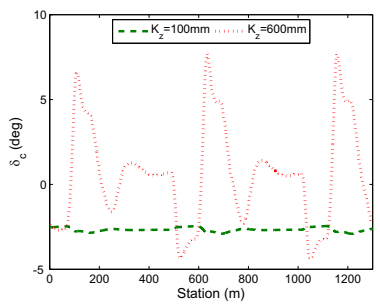

(f) Front left wheel steering
Fig. 6. Simulation results in vehicle maneuvers

exceeds $1.5 \mathrm{~m}$, which may cause lane departures. Using the variable-geometry control system as a driver assistance system the error is reduced significantly, which is shown in Figure 6(b). Note that the reduction of the lateral error is independent of $K_{z}$, it is based on the designed controller.

The half-track change of the suspension system is shown in Figure 6(c). If $K_{z}=100 \mathrm{~mm}$ construction is set, in general, the half-track change is better than in the case of $K_{z}=600 \mathrm{~mm}$. However, the peak value of the half-track 
change is significantly worse in the $K_{z}=100 \mathrm{~mm}$ case. Besides, the actuation of control systems is greater in the $K_{z}=100 \mathrm{~mm}$ construction, see Figure 6(d). Generally, the tendencies of control input signals are the same in both constructions. An interaction between $\Delta B$ and $a_{y}$ is also found. When the $K_{z}=600 \mathrm{~mm}$ construction is set the peak values of signal $a_{y}$ increase compared to the construction $K_{z}=100 \mathrm{~mm}$.

In terms of $\gamma$ and $\delta_{c}$ the effects of the suspension constructions are different. In the case of $K_{z}=100 \mathrm{~mm}$ the control system is able to affect mainly the modification of wheel camber angle $\gamma$, see Figure 6(e). $\gamma$ values are higher than in the other case because this system guarantees trajectory tracking by modifying $\gamma$. When $K_{z}=600 \mathrm{~mm}$ the control system is able to affect both wheel camber angle $\gamma$ and steering angle $\delta_{c}$, see also Figure 6(f). Since in this suspension system the steering wheel angle cooperates with the wheel camber angle, a reduced $a_{y}$ actuation is sufficient to perform trajectory tracking.

The operation of low-level actuator control is shown in Figure 7. The signal of the high-level control $a_{y}$ is the

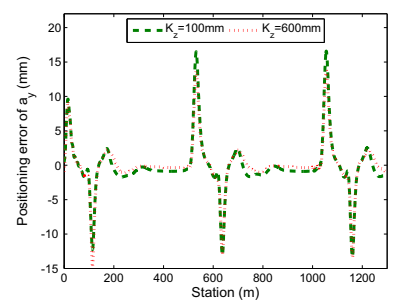

(a) Tracking error of signal $a_{y}$

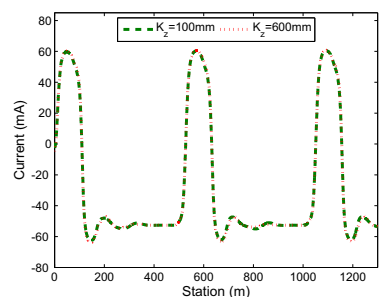

(b) Current $i$

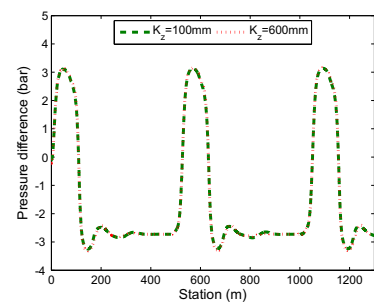

(c) Pressure $p_{L}$

Fig. 7. Operation of the actuator control

requirement of the lateral displacement, see Figure 6(d). This signal is realized by the electro-hydraulic actuator based on the $\mathcal{H}_{\infty} / \mu$ method.

It is shown that the actuator is able to reduce the tracking error below an appropriate value, see Figure 7(a). The designed control is able to hold the current of the spool valve in an acceptable range as Figure 7(b) shows. The pressure difference $p_{L}$ of the chamber is depicted in Figure 7(c). It can be stated that the designed low-level control is able to generate the required control input of the high-level control $a_{y}$.

\section{CONCLUSION}

The paper has proposed a two-step procedure for the design of a variable-geometry suspension system. In the high-level control various vehicle performances must be guaranteed such as trajectory tracking, the reduction of the chassis roll angle and the minimization of half-track change. In the low-level control the actuator must track this signal by adjusting its current signal. The advantage of this modular design is that the actuator level does not affect the design of a high-level control. The simulation example illustrates the efficiency of the variable-geometry suspension system and it shows that the system is suitable to be used as a driver assistance system.

\section{REFERENCES}

[1] W. Evers, A. van der Knaap, I. Besselink, and H. Nijmeijer, "Analysis of a variable geometry active suspension," International Symposium on Advanced Vehicle Control, Kobe, Japan, pp. 1-6, 2008.

[2] R. Sharp, "Variable geometry active suspension for cars," IEEE Computing and Control Engineering Journal, vol. 9, no. 5, pp. 217 222, 1998.

[3] M. S. Fallah, R. Bhat, and W. F. Xie, "New model and simulation of McPherson suspension system for ride control applications," Vehicle System Dynamics, vol. 47, no. 2, pp. 195-220, 2009.

[4] M. Vukobratovic and V. Potkonjak, "Modeling and control of active systems with variable geometry. i: General approach and its application," Mechanism and Machine Theory, vol. 35, pp. 179-195, 1999.

[5] U. K. Lee, S. H. Lee, C. S. Han, K. Hedrick, and A. Catala, "Active geometry control suspension system for the enhancement of vehicle stability," Proceedings of the IMechE, Part D: Journal of Automobile Engineering, vol. 222, no. 6, pp. 979-988, 2008.

[6] B. Németh and P. Gáspár, "Mechanical analysis and control design of McPherson suspension," International Journal of Vehicle Systems Modelling and Testing, vol. 7, no. 2, pp. 173-193, 2012.

[7] H. B. Pacejka, Tyre and vehicle dynamics. Oxford: Elsevier Butterworth-Heinemann, 2004.

[8] B. Németh and P. Gáspár, "Integration of control design and variable geometry suspension construction for vehicle stability enhancement," Proc. of the Conference on Decision and Control, Orlando, Fl, pp. 1-6, 2011.

[9] V. Gough and G. Shearer, "Front suspension and tyre wear," The Institution of Mechanical Engineers, Proceedings of the Automobile Division, pp. 171-216, 1956.

[10] J. Bokor and G. Balas, "Linear parameter varying systems: A geometric theory and applications," 16th IFAC World Congress, Prague, pp. $1-6,2005$.

[11] A. Packard and G. Balas, "Theory and application of linear parameter varying control techniques," American Control Conference, Workshop I, Albuquerque, pp. 1-6, 1997.

[12] I. Esfahani, M. Mosayebi, M. Pourshams, and A. Keshavarzi, “Optimization of double wishbone suspension system with variable camber angle by hydraulic mechanism," Engineering and Technology, pp. 299-306, 2010

[13] S. Lee, H. Sung, J. Kim, and U. Lee, "Enhancement of vehicle stability by active geometry control suspension system," International Journal of Automotive Technology, vol. 7, no. 3, pp. 303-307, 2006.

[14] B. Sulc and J. A. Jan, "Non linear modelling and control of hydraulic actuators," Acta Polytechnica, vol. 42, no. 3, pp. 41-47, 2002.

[15] M. Karpenko and N.Shapehri, "Fault-tolerant control of a servohydraulic positioning system with crossport leakage," IEEE Transactions on Control Systems Technology, vol. 13, pp. 155-161, 2005.

[16] F. Wijnheijmer, G. Naus, W. Post, M. Steinbuch, and P. Teerhuis, "Modelling and LPV control of an electro-hydraulic servo system," IEEE Computer Aided Control System Design, Münich, 2006.

[17] H. E. Meritt, Hydraulic control systems. New York: John Wiley \& Sons, Inc., 1967.

[18] A. Packard and J. Doyle, "The complex structured singular value," Automatica, vol. 29, no. 1, pp. 71-109, 1993.

[19] P. Young, M. Newlin, and J. Doyle, " $\mu$ analysis with real parametric uncertainty," Proc. of the Conference on Decision and Control, Brighton, England, pp. 1251-1256, 1991. 